\title{
Premature ejaculation among post-coronary artery stenting patients attending cardiology out patient department of tertiary care hospitals
}

\author{
Shahriar Faruque, ${ }^{1}$ Salahuddin Qusar Biplob, ${ }^{2}$ Mekhala Sarkar, ${ }^{3}$ Nafia Farzana, ${ }^{4}$ Wasima Rahman, ${ }^{5}$ Fatima Zohra, ${ }^{6}$ \\ Mohammad Waliul Hasnat Sajib ${ }^{7}$ \\ ${ }^{1}$ Registrar, Department of Psychiatry, Shaheed Ziaur Rahman Medical College, Bogura, Bangladesh; ${ }^{2}$ Professor \& Head, Department \\ of Psychiatry, Bangabandhu Sheikh Mujib Medical University (BSMMU), Dhaka, Bangladesh; ${ }^{3}$ Associate Professor (Psychiatry), \\ National Institute of Mental Health, Dhaka, Bangladesh; ${ }^{4}$ Medical Officer, Department of Psychiatry, BSMMU, Bangladesh; ${ }^{5}$ Medical \\ Officer, Department of Psychiatry, BSMMU, Bangladesh; ${ }^{6}$ Assistant Professor, Department of Psychiatry, Uttara Adhunik Medical \\ College, Dhaka, Bangladesh; ${ }^{7}$ Assistant Professor, Department of Psychiatry, Shaheed M Monsur Ali Medical College, Sirajgonj, \\ Bangladesh.
}

$\begin{array}{ll}\text { Article info } & \\ \text { Received } & : \text { 10 Jan, } 2020 \\ \text { Accepted } & : 05 \text { May, 2020 } \\ \text { Number of tables } & : 04 \\ \text { Number of figures } & : 00 \\ \text { Number of refs } & : 20\end{array}$

Correspondence

Shahriar Faruque

Mobile: 01712045011

E-mail:anikmmc@gmail.com

\begin{abstract}
Summary
Premature ejaculation (PE) is the most frequent sexual complaint of men. It may develop not only due to psychiatric disorders but also due to many chronic organic illnesses. Coronary artery stenting is an invasive procedure to manage coronary artery disease which is one of the leading chronic illnesses all over the world. Patients who survive from coronary artery diseases by getting invasive cardiac procedures, often develop reduced sexual function. The aim of the study was to identify the frequency of premature ejaculation among post coronary artery stenting patients. This was a descriptive, cross sectional study conducted in cardiology outpatient department (OPD) of Bangabandhu Sheikh Mujib Medical University (BSMMU) and cardiology OPD of National Institute of Cardiovascular Diseases (NICVD) from October 2017 to September 2018. 153 patients were selected purposively. After taking written consent a predetermined questionnaire containing 11 socio-demographic variables was filled for each patient through face to face interview. After that PEDT bangle version was applied. $37.25 \%$ post coronary artery stenting male scored 11 or more and were identified as highly suggestive to have PE. Among the socio-demographic variables, Regular exercise, amount of sleep per day, smoking and history of chronic physical illness were found to be significantly associated with PE among post coronary artery stenting patients. The study showed that the patients who practiced a disciplined and healthy lifestyle were not in risk. The research findings may help us for the early diagnosis and better treatment plan to reduce patient sufferings.
\end{abstract}

Bang J Psychiatry 2018;32(1): 9-13

\section{Introduction}

Premature ejaculation (PE) is likely the most common sexual dysfunction in men. It has a worldwide prevalence of approximately $30 \% .{ }^{1}$ A study conducted in Asia-pacific region reveals that the prevalence of $\mathrm{PE}$ in that region is $31 \%{ }^{2}$ According to an internet based premature ejaculation prevalence and attitude (PEPA) survey, prevalence of PE is $24.0 \%$ in the United States, $20.3 \%$ in Germany, and $20.0 \%$ in Italy. ${ }^{3}$ According to a study published in August, 2016 the rate of acquired PE among Turkish population is $3.9 \%$ and among Chinese population it is $4.5 \% .{ }^{4}$ The above mentioned studies have shown the prevalence of PE which comprises both the life-long and acquired PE. According to Diagnostic and Statistical Manual, version four (DSM-4), premature ejaculation is defined as a male sexual dysfunction characterized by: (A) a persistent or recurrent ejaculation with minimal sexual stimulation before, on or shortly after penetration and before the person wishes it, $(B)$ the symptom causes marked distress or interpersonal difficulty, (C) the symptom is not due to direct effect of a substance. ${ }^{5}$ Lack of ejaculatory control results in greater dissatisfaction and greater emotional distress, including poor self-esteem, feelings of inadequacy, disappointment, and anxiety. Thus, continued PE ultimately leads to greater problems with partner and often disrupts partner relationship. ${ }^{6}$

Though premature ejaculation has a widespread and significant negative impact on a person's life, there is very few comprehensive data regarding its etiology. Previously, PE was 
considered only a psychological problem and as a neurosis which is linked to an unconscious conflicts. ${ }^{7}$ But at present, evidence based studies have identified that although psychogenic causes such as performance anxiety remain important underlying conditions of $\mathrm{PE}^{8}$, in some cases, chronic organic factors have been suggested as important etiology of PE. ${ }^{9}$ Different neurotransmitters and hormones have roles in male ejaculation. Evidence suggests that some neurotransmitters like norepinephrine, serotonin, oxytocin, gamma-amino-butyric acid, and nitric oxide and sex hormone estrogen play central roles in ejaculation. These agents subsequently may mediate $\mathrm{PE} .{ }^{10}$ On the other hand, increased level of serum estrogen is strongly associated with unfavorable lipid profile in early life of males. ${ }^{11}$ And this is now an established fact that abnormal lipid profile is one of the most important risk factors to develop coronary heart diseases.

Due to the modern and sedentary life style, people of the developed as well as developing countries are more vulnerable to develop different chronic organic illnesses such as coronary artery disease, diabetes mellitus, chronic kidney disease etc. A data published in 2014 by Center for Disease Control and prevention shows that coronary heart disease is one of the top 10 leading causes $\left(4^{\text {th }}\right)$ of death in Bangladesh and it accounts for $6.4 \%$ of total death in a year. ${ }^{12}$ According to World health organization (WHO) 2017, about 7.4 million deaths occurred in 2016 worldwide due to coronary artery disease. ${ }^{13} \mathrm{~A}$ review over 40 year's literatures has shown that there is significant evidence of the negative impact of coronary heart disease on sexual activity. ${ }^{14}$ Cardiovascular diseases affect patient's sexual activity for two primary reasons: 1 ) diagnosis of a heart condition and all the psychological implications such as anxiety, fear of death and restricted physical activity, 2) they need to use several drugs capable of producing adverse effects that compromise sexual performance. ${ }^{15}$

Management of coronary artery disease patients includes a large variety of agents and procedures that are invasive in varying degrees. These procedures produce different levels of restriction to physical activities which ultimately leave an impact on patient's sexual life. Coronary artery stenting and coronary artery bypass graft surgery are the two invasive options for treating coronary artery disease. Patients who survive with coronary artery disease followed by an invasive procedure often develop decreased sexual functions, anxiety, depression and other psychiatric co-morbidities. A large number of patients won't be able to resume their normal sexual activity after a cardiac event and invasive procedure. Compared to the preoperative state, there was a significant deterioration of sexual functions in patients after coronary artery bypass graft (CABG) surgery and the impairment was evident in elements of ejaculation, erection, sexual excitement, and enjoyment and satisfaction. ${ }^{16}$ Following a cardiac diagnosis and procedure, it has been estimated that about $25 \%$ of the patients resume their normal sexual activity, with the previous frequency and intensity, half of the patients return to their sexual activity at a reduced level and the other $25 \%$ do not resume any sexual activity at all. ${ }^{15}$

Physicians need to address patient's sexual problems during cardiac rehabilitation process. Many patients and their partners report that sexuality remains as an important activity, which is rarely addressed by health professionals. ${ }^{17}$ In perspective of Bangladesh, there is a substantial lack of scientific papers to show the proportion of premature ejaculation in patients who have got coronary artery stenting. Some international papers show the relation between coronary artery bypass graft surgery and sexual dysfunctions but there is lack of data to identify the relation between sexual dysfunction and cardiac intervention procedure such as coronary artery stenting. Moreover, stenting in coronary artery as a management of coronary artery disease is becoming more popular now a day as it is less invasive than CABG. The present study was designed with the main goal to obtain credible baseline data to identify the proportion of premature ejaculation among the patients who have got coronary artery stenting after coronary artery disease. Moreover, it was intended to find out the socio-demographic correlates of premature ejaculation after an invasive cardiac procedure like stenting. This study will influence the clinicians to make early diagnosis and to start early management in clinical setting and during cardiac rehabilitation. Overall, this may help to reduce the burden of life of the patient.

\section{Materials and methods}

This was a cross sectional study done at cardiology outpatient departments of Bangabandhu Sheikh Mujib Medical University, Dhaka, Bangladesh and National Institute of Cardiovascular Diseases, Dhaka, Bangladesh from October 2017 to September 2018. Total 153 patients who were married, got coronary artery stenting at least 6 months before the interview and had active sexual life after coronary artery stenting were selected purposively and were interviewed. Patients aged over 60 years of age were avoided as sexual dysfunctions are usually common in old ages. The patients who had history of premature ejaculation prior to coronary artery stenting were excluded from the study. The patients were from different ages ranged from 32 years to 60 years. The patients were informed about the purpose of the study and ethical issues. Then after taking the written informed 
consent, data collection procedure was initiated by the researcher maintaining best possible ways to ensure privacy of the patients. Data was collected through face to face interview using the questionnaire for the study of premature ejaculation among postcoronary artery stenting patients. After that PEDT bangle version was applied and a score was generated for each patient according to the scoring system of the tool. PEDT is a 5-items, uni-dimensional measure, which contains the essence of DSMIV-TR: control, frequency, minimal stimulation, distress, and interpersonal difficulty. A score of d" 8 indicated no PE, a score of 9 and 10 suggests borderline or probable for $P E$, and e" 11 suggests highly suggestive for PE.Data were processed and analyzed using SPSS (Statistical Package for Social Sciences), version 24.0. The categorical data were expressed as frequency and percentage and were compared among variables using cross-tabulation and Chi-square $\left(C^{2}\right)$ Test. The level of significance was set at $5 \%$ and $p$-value $<0.05$ was considered significant.

\section{Results}

In this study, among 153 patients, $37.25 \%$ (57 out of 153) post coronary artery stenting male were found as highly suggestive to have premature ejaculation. Another $62.75 \%$ (96 out of 153) were borderline and non-suggestive to have PE (Table 1).

Association of premature ejaculation with socio-demographic variables found that, different age range $(p=0.098)$, education $(p=0.529)$, profession $(p=0.861)$ and residence $(p=0.732)$ were not found significantly associated with PE among the post coronary artery stenting patients (Table 2 ). Among the factors related to lifestyle, regular physical exercise $(p=0.000)$, amount of sleep per day $(p=0.000)$, smoking $(p=0.011)$ and regular medication taking $(p=0.006)$ were found to be significantly associated with $\mathrm{PE}$ among post coronary artery stenting patients but history of substance abuse $(p=0.146)$ was not found to be significantly associated (Table 3 ).

On the other hand, regarding history of previous illnesses, history of chronic physical illness $(p=0.000)$ was significantly associated with $\mathrm{PE}$ among post-coronary artery stenting patients but history of previous psychiatric illness $(p=0.529)$ found not to be associated (Table 4).

Table 1: Frequency of PE among post coronary artery stenting patients $(n=153)$.

\begin{tabular}{lcc}
\hline & $\begin{array}{c}\text { Number of } \\
\text { patients }\end{array}$ & Percent \\
\hline Highly suggestive & 57 & $37.25 \%$ \\
Borderline and not suggestive for PE & 96 & $62.75 \%$ \\
\hline
\end{tabular}

Table 2: Association between socio-demographic variables and post coronary artery stenting patients with premature ejaculation $(n=153)$.

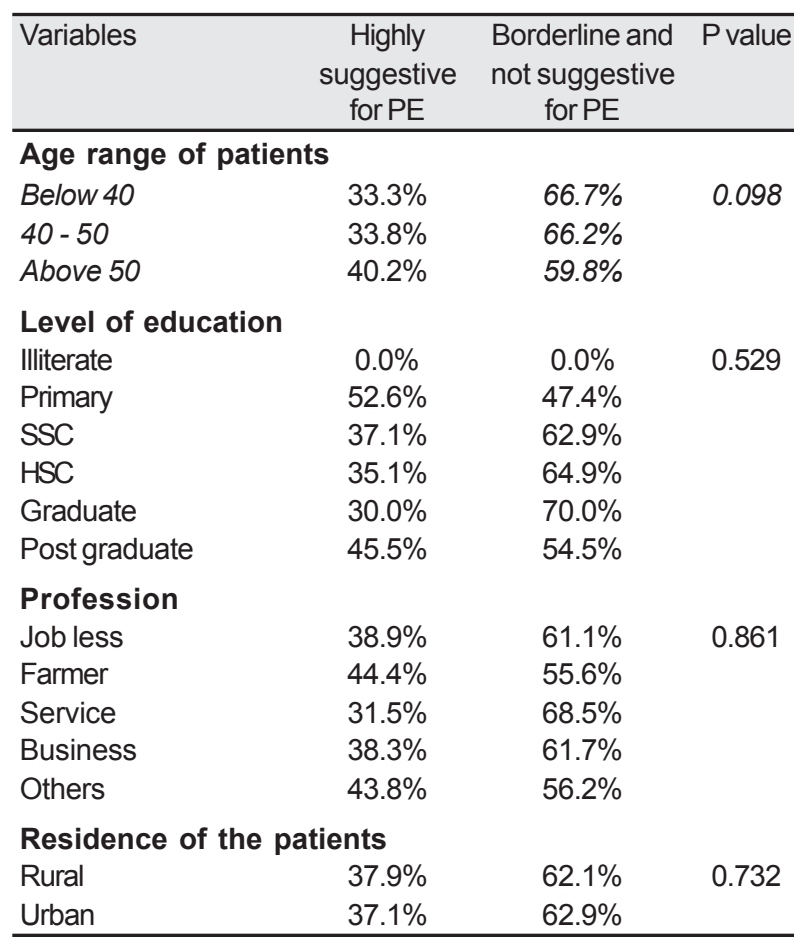

Table 3: Association between life events and post coronary artery stenting patients with premature ejaculation $(n=153)$.

\begin{tabular}{|c|c|c|c|}
\hline Life Style & $\begin{array}{c}\text { Highly } \\
\text { suggestive } \\
\text { for PE }\end{array}$ & $\begin{array}{c}\text { Borderline and } \\
\text { not suggestive } \\
\text { for PE }\end{array}$ & Pvalue \\
\hline \multicolumn{4}{|c|}{ Daily Activities (No. Of Days) } \\
\hline None & $75.5 \%$ & $24.5 \%$ & 0.000 \\
\hline 3 days a week or less & $25.8 \%$ & $74.2 \%$ & \\
\hline 4 days a week or more & $9.5 \%$ & $90.5 \%$ & \\
\hline \multicolumn{4}{|l|}{ Sleep (No. of Hours) } \\
\hline Less than 6 hours & $61.9 \%$ & $38.1 \%$ & 0.000 \\
\hline 6 to 8 hours & $19.6 \%$ & $80.4 \%$ & \\
\hline More than 8 hours & $14.3 \%$ & $85.7 \%$ & \\
\hline \multicolumn{4}{|l|}{ H/O Smoking } \\
\hline Present & $52.2 \%$ & $47.8 \%$ & 0.011 \\
\hline Absent & $30.8 \%$ & $69.2 \%$ & \\
\hline \multicolumn{4}{|c|}{ Regular medication for IHD } \\
\hline Yes & $32.8 \%$ & $67.2 \%$ & 0.006 \\
\hline No & $63.6 \%$ & $36.4 \%$ & \\
\hline \multicolumn{4}{|l|}{ H/O Substance abuse } \\
\hline Present & $75.0 \%$ & $25.0 \%$ & 0.146 \\
\hline Absent & $36.2 \%$ & $63.8 \%$ & \\
\hline
\end{tabular}


Table 4: Association between history of illness and post coronary artery stenting patients with premature ejaculation $(n=153)$.

\begin{tabular}{lccc}
\hline Illness & $\begin{array}{c}\text { Highly } \\
\text { suggestive } \\
\text { for PE }\end{array}$ & $\begin{array}{c}\text { Borderline and } \\
\text { not suggestive } \\
\text { for PE }\end{array}$ & P value \\
\hline H/O Chronic Physical IIIness & & \\
Present & $61.2 \%$ & $38.8 \%$ & 0.000 \\
Absent & $11.0 \%$ & $89.0 \%$ & \\
H/O Previous Psychiatric IIIness & & \\
Present & $0.0 \%$ & $100 \%$ & 0.529 \\
Absent & $37.7 \% \%$ & $62.3 \%$ & \\
\hline
\end{tabular}

\section{Discussion}

Premature ejaculation is most commonly found sexual disorder in male. In this study, $37.25 \%$ post coronary artery stenting male were found as highly suggestive to have PE and another $7.20 \%$ were borderline to have PE. It was found that many scientific studies were conducted to find the frequency of sexual dysfunction as a whole or to find the frequency of erectile dysfunction after coronary artery bypass graft surgery. According to a study conducted in Egypt, there was a significant deterioration of sexual functions in patients after CABG surgery compared to the preoperative state. The impairments were evident in elements of ejaculation, erection, sexual excitement, and enjoyment and satisfaction. That study also showed that among 54 patients, no one had any complaint of premature ejaculation but after CABG surgery 28 (51.9\%) of them complained about premature ejaculation which was higher than that in our study. ${ }^{16}$ In our country many men are reluctant to seek professional advice and therefore resort to self-treatment for extended period. So that the frequency was less in our study but we think that the number was still high.

Four socio-demographic variables, history of previous illness and factors associated with lifestyle were analyzed to find if they had any significant association in premature ejaculation among post coronary artery stenting patients. Among the sociodemographic variables taken in this study, age of the patients, education of the patients, profession of the patients and residence of the patients were not found to be significantly associated with premature ejaculation among post-coronary artery stenting patients. Among the factors related to lifestyle and history of previous illness, history of previous psychiatric illness and history of substance abuse were not found to be significantly associated with PE among post-coronary artery stenting patients. On the other hand, amount of sleep per day, regular physical exercise, history of smoking, history of chronic physical illness and regular cardiac medication were found to have significant association with PE among post coronary artery stenting patients. According to PEDT, the post-coronary artery stenting patients who did not use to take regular physical exercise were more suggestive to have PE than the patients who took regular physical exercise. This finding can be correlated with the finding of the study done by Elizabeth Anderson that regular physical exercise and regular activity positively impacts the pathophysiological processes of anxiety and physical activity positively impacts a number of biological, as well as psychological mechanisms as anxiety is an important cause of PE. ${ }^{18}$ Similarly, the post-coronary artery stenting patients who had less than 6 hours of sleep per day were more suggestive to have PE in comparison to the patients who had 6 to 8 hours of sleep per day. Insomnia is a feature of anxiety. So, the finding of this study also co-related with the finding of the study done by K. M. Dunn in 1999 which revealed that premature ejaculation is predominantly associated with anxiety. ${ }^{19}$ Chronic illness and its treatments can have a negative impact on sexual functioning. ${ }^{20}$ In this study, patients with chronic physical illness such as diabetes mellitus, hypertension, abnormal thyroid function etc. were more suggestive to have PE than the patients who had no history of such chronic physical illness. So, this finding matched with finding of previous study on a similar topic. Post- coronary artery stenting patients who used to smoke, were more suggestive to have PE than the patients who did not smoke. Regarding regular medication, it is observed that the patients who took medication regularly for their cardiac problems, were less suggestive to have PE.

In a developing country like Bangladesh, there are so many myths about invasive cardiac procedures and also about sexual dysfunctions. Invasive cardiac procedure or stenting reduces sexual functions - is one of the most common myths. Though in this study, it was found that the frequency of acquired premature ejaculation is much higher among post-coronary artery stenting patients but due to findings other variables it cannot be concluded that coronary artery stenting itself had any direct significant association in developing premature ejaculation. The study findings showed that after coronary artery stenting, the patients who used to take regular physical exercise, used to take adequate sleep and gave up smoking were less likely to develop premature ejaculation. This indicates that after having coronary artery stenting if a patient practices a healthy and disciplined lifestyle can have a healthy sexual life as well as a quality life.

It was a hospital based cross sectional study and researchers did the study in two hospitals in Dhaka city. So, it did not represent the whole group of such patients. Further research should be aimed to include a larger sample size selected from a larger number of different cardiac and multi-disciplinary hospitals of different parts of the country. 


\section{Conclusion}

Patients with coronary artery stenting are vulnerable for PE but, who practice a disciplined and healthy lifestyle, are not in risk. On the other hand, the patients who do not practice a disciplined and healthy lifestyle like, do not take adequate sleep, do not have physical exercise, continue smoking and do not take regular medication for their illness, are in a risk to have PE. So, at the time of discharge from hospital after coronary artery stenting, a patient should be counseled well regarding the necessity to follow a healthy lifestyle. During follow up, it is important to address this issue when patients come to cardiologist so that, proper management plan can be made in collaboration with a psychiatrist.

\section{References}

1. Carson C and Gunn K. Premature ejaculation: definition and prevalence.Int J Impot Res 2006;18:S5-13.

2. McMahon CG, Lee G, Park JK andAdaikan PG. Premature Ejaculation and Erectile Dysfunction Prevalence and Attitudes in the Asia-Pacific Region. J Sex Med 2006;9(2):454-65.

3. Porst H, Montorsi F, Rosen RC, Gaynor L, Grupe S and Alexander J. The Premature Ejaculation Prevalence and Attitudes (PEPA) survey: prevalence, comorbidities, and professional help-seeking. European Urology 2007;51(3):816-23.

4. Saitz, T. and Serefoglu, E. (2016). The epidemiology of premature ejaculation. Transl Androl Urol 2016;5(4): 409-15.

5. Diagnostic and Statistical Manual of Mental Disorder (DSM-IV). 4th edition. American Psychiatric Association 1994.

6. Revicki D, Howard K, Hanlon J, Mannix S, Greene $A$ and Rothman M. Characterizing the burden of premature ejaculation from a patient and partner perspective: a multi-country qualitative analysis. Health Qual Life Outcomes 2008;6:33.

7. Jannini E, Simonelli C and Lenzi A. Sexological approach to ejaculatory dysfunction. Int J Androl 2002;25:317-23.
8. Althof SE, McMahon CG, Waldinger MD, Serefoglu EC, Shindel AW, AdaikanPGet al. An update of the International Society of Sexual Medicine's guidelines for the diagnosis and treatment of premature ejaculation (PE). J Sex Med 2014;11:1392-422.

9. Waldinger MD. Ejaculatio praecox, erectio praecox and detumescentia praecox as symptoms of a hypertonic state in lifelong premature ejaculation: A new hypothesis. PharmacolBiochemBehav 2014;121:189-94.

10. DonatucciCF. Etiology of Ejaculation and Pathophysiology of Premature Ejaculation. J Sex Med 2006;3(4):303-08.

11. Tomaszewski M, Charchar FJ, Maric C, Kuzniewicz R, Gola M, Grzeszczak W et al. Age and psychologic disorders. Variables associated to post- infarction sexual dysfunction. Arq Bras Cardiol 2005;85(2):110-4.

12. CDC Global Health - Bangladesh - Center for Disease Control and Prevention. https://www.cdc.gov/ globalhealth/countries/bangladesh/default.htm

13. World Health Organization (2017), http://www.who.int/ mediacentre/factsheets/fs317/en/.

14. Weiss E, Dlin B, Rollin H, Fischer $\mathrm{H}$ and Bepler C. Emotional factors in coronary occlusion. Arch Intern Med 1957;99:628-41.

15. Thorson A. Sexual activity and the cardiac patient. Am J Geriatr Cardiol 2003;12:38-40.

16. Mourad F, Mohamed El, Ahmad EM, Walaa S and Mostafa B. Sexual dysfunction before and after coronary artery bypass graft surgery in males. Journal of the Egyptian Society of Cardio-Thorasic Surgery 2017;25(10):45-51.

17. Friedman S. Cardiac disease, anxiety and sexual functioning.Am J Cardiol 2000;86(2):46-50.

18. Anderson E \&Shivakumar G. Effects of Exercise and Physical Activity on Anxiety. Front Psychiatry 2013;4:27.

19. Dunn KM, Croft PR and Hackett GI. Association of sexual problems with social, psychological, and physical problems in men and women: a cross sectional population survey. J Epidemiol Community Health 1999; 53:144-48.

20. Nusbaum MR, Hamilton C, Lenahan P. Chronic Illness and Sexual Functioning. Am Fam Physician 2003;67(2): 347-54. 